\title{
Erratum
}

\section{A generalisation of the theory of geometrical shock dynamics}

J.P. Best

Materials Research Laboratory (MRL) - DSTO, P.O. Box 50, Melbourne 3032, Australia

Shock Waves 1, 251-273 (1991)

In Best (1991), equation (173) should read

$$
\begin{aligned}
\delta \xi(t & +\delta t) \approx\left(\left|\frac{\partial \mathbf{x}(\xi(t), t)}{\partial \xi(t)}\right|^{2}+2 a_{0} \frac{\partial \mathbf{x}(\xi(t), t)}{\partial \xi(t)}\right. \\
\cdot & \left(M(\xi(t), t) \frac{\partial \mathbf{n}(\xi(t), t)}{\partial \xi(t)}\right. \\
& \left.\left.+\frac{\partial M(\xi(t), t)}{\partial \xi(t)} \mathbf{n}(\xi(t), t)\right) \delta t\right)^{\frac{1}{2}} \delta \xi(t) \\
& \approx\left(1+a_{0} M(\xi(t), t) \frac{\partial \mathbf{x}(\xi(t), t)}{\partial \xi(t)}\right. \\
& \left.\cdot \frac{\partial \mathbf{n}(\xi(t), t)}{\partial \xi(t)} \delta t\right) \delta \xi(t)
\end{aligned}
$$

and equation (175) should read

$$
\begin{aligned}
\frac{\delta \xi(t+\delta t)-\delta \xi(t)}{\delta \xi(t) \delta t} \approx & a_{0} M(\xi(t), t) \frac{\partial \mathbf{x}(\xi(t), t)}{\partial \xi(t)} \\
& \cdot \frac{\partial \mathbf{n}(\xi(t), t)}{\partial \xi(t)}
\end{aligned}
$$

The reference to the paper by Lighthill should read as set out below.

\section{References}

Best JP (1991) A generalisation of the theory of geometrical shock dynamics. Shock Waves 1(4):251-273

Lighthill MJ (1949) The diffraction of blast I. Proc Roy Soc London A $198: 454-470$ 